\title{
Experimental studies of electron transport
}

\author{
F Ryter ${ }^{1}$, C Angioni ${ }^{2}$, M Beurskens ${ }^{3}$, S Cirant ${ }^{4}$, G T Hoang ${ }^{5}$, \\ G M D Hogeweij ${ }^{3}$, F Imbeaux ${ }^{5}$, A Jacchia ${ }^{4}$, P Mantica ${ }^{4}$, W Suttrop ${ }^{1}$ and \\ G Tardini ${ }^{1}$
}

${ }^{1}$ Max-Planck-Institut für Plasmaphysik, EURATOM Association, D-85748 Garching, Germany

${ }^{2}$ Centre de Recherches en Physique des Plasmas, Ecole Polytechnique de Lausanne, Association EURATOM-Confédération Suisse, CH-1015 Lausanne, Switzerland

${ }^{3}$ FOM Institut voor Plasmafysica 'Rijnhuizen', Associate Euratom-FOM,

Trilateral Euroregio Cluster, PO Box 1207, 3430 BE Nieuwegein, The Netherlands

${ }^{4}$ Istituto di Fisica del Plasma, Associazione EURATOM-ENEA-CNR, 20133 Milano, Italy

${ }^{5}$ Département de Recherches sur la Fusion Contrôlée, Association Euratom-CEA,

CEA Cadarache, 13108 St Paul lez Durance Cedex, France

Received 22 June 2001

Published 22 November 2001

Online at stacks.iop.org/PPCF/43/A323

\begin{abstract}
Electron transport in tokamaks has many different features which are briefly reviewed. The paper is focused on electron heat transport in conventional tokamak plasmas. An inter-machine comparison indicates that the nondimensional gradient length of the electron temperature profiles $R / L_{T_{\mathrm{e}}}$ is almost independent of the devices and varies little with plasma parameters. This strongly suggests that electron heat transport is governed by turbulence with a threshold in $R / L_{T_{\mathrm{e}}}$. This is confirmed by modulation experiments using electron cyclotron heating. Simulations with empirical and physics-based transport models confirm this assumption.
\end{abstract}

\section{Introduction}

Understanding transport in fusion experiments, through both electron and ion channels, is essential for planning and predicting future devices. In this paper we concentrate on the electron heat channel in tokamaks which is larger than the neoclassical prediction by about two orders of magnitude. This is attributed to micro-turbulence.

However, the electron temperature profiles can exhibit quite different behaviours depending on the experimental conditions, which probably reflects various physics mechanisms, not all of them being related to turbulent transport. They can be classified as follows.

- 'Profile resilience': an early-recognized feature, first observed in ohmically heated plasmas and extensively studied later with auxiliary heating and still of actuality as we will see. This seems to be a quite general behaviour in tokamaks. 
- Transport barriers around rational $q$ surfaces have been reported and indeed the $q=1$ surface seems to play a particular role in this respect. In most of the cases, such barriers can be assigned to the reduction or suppression of the turbulent transport related to flat or reversed magnetic shear.

- 'Non-local transport': this term is applied to a phenomenon in which a fast response to an edge perturbation is observed in the centre, generally with inverse polarity. The phenomenon is generally observed at very low density and it is not yet clear how it is related to the transport observed in the usual density range.

- 'Filaments': fast time-varying structures on a millimetre scale in the radial direction, generally appearing inside the $q=1$ surface, have been observed in conjunction with strong central electron heating.

Finally, in both stellarators and tokamaks, a strong correlation between heat and particle transport is observed.

In this paper, which is not meant to be an exhaustive review of experimental investigations on electron transport, we essentially report on recent developments in this field. We also restrict comment to electron transport in conventional scenarios ( $\mathrm{L}$ and $\mathrm{H}$ modes) and do not include internal transport barriers (ITBs). This topic is treated in a companion paper at this conference [1]. Finally, we emphasize the aspects of electron transport which appear to be linked with turbulent transport. The motivation for this is that it seems essential for extrapolation to future devices and can now be compared to transport models based on first-principles turbulence calculations.

In the next section we first provide some background on electron transport studies. The main experimental results on electron transport are summarized in sections 3 and 4 . In section 5 these results are compared with modelling; section 6 is dedicated to discussion and conclusions.

\section{Physical background}

\subsection{Transport theory}

The most probable candidates believed to cause turbulent transport in tokamaks are the trapped electron (TEM) modes, generally associated with the ion temperature gradient (ITG) modes, and the electron temperature gradient modes (ETG) [2,3]. These basic instabilities have been known for a long time [4], but only for a few years have numerical calculations based on these principles provided transport models which can be directly and conveniently compared with the experimental results. The case of ETG is somewhat particular; due to the very small size of the turbulence cells, significant transport was originally not expected to be driven. Very recently, nonlinear calculations indicated that ETG modes may create cells with large radial extension and are therefore indeed able to drive transport comparable with the experimentally measured values $[5,6]$. The TEM and ETG instabilities have the essential common property of developing above a threshold in $\nabla T_{\mathrm{e}} / T_{\mathrm{e}}$. The values and parameter dependences of the respective thresholds are different. These thresholds are predicted to vary with plasma parameters: $\nabla n_{\mathrm{e}} / n_{\mathrm{e}}$ and the fraction of trapped electrons for TEMs [7]; $T_{\mathrm{e}} / T_{\mathrm{i}}$, effective ion charge $Z_{\text {eff }}$ and ratio of magnetic shear to safety factor $\hat{s} / q$, or $\nabla n_{\mathrm{e}} / n_{\mathrm{e}}$ for peaked density profiles, in the case of ETG $[3,8]$.

This means that turbulence and therefore transport increases locally as soon as the temperature profiles exceed $(\nabla T / T)_{\mathrm{c}}$ at any radial location. This tends to prevent the profiles from increasing above the critical value, more or less vigorously depending on how the driven transport increases with $\nabla T / T$ above the threshold. If the instability (and transport) grows very strongly just above the threshold, the profiles will be kept quite close to $(\nabla T / T)_{\mathrm{c}}$, leading 
to 'stiff' transport behaviour or 'stiff profiles'. On the contrary, if the transport driven by the instability increases weakly above the threshold, the experimental $\nabla T / T$ values may be significantly above $(\nabla T / T)_{\mathrm{c}}$.

A simple model for the heat diffusivity $\chi_{\mathrm{e}}$ determined by temperature gradient $(\mathrm{TG})$ driven turbulence can be written as

$$
\chi_{\mathrm{e}}=T_{\mathrm{e}}^{3 / 2}\left[\xi_{0}+\mathcal{G}\left(\nabla T_{\mathrm{e}} / T_{\mathrm{e}}-\left(\nabla T_{\mathrm{e}} / T_{\mathrm{e}}\right)_{\mathrm{c}}\right)\right]
$$

where $\xi_{0}$ describes the transport in the absence of TG modes and might be as low as neoclassical transport. The function $\mathcal{G}$, which describes the transport driven by $\mathrm{TG}$, equals zero below $\left(\nabla T_{\mathrm{e}} / T_{\mathrm{e}}\right)_{\mathrm{c}}$ and increases above it. Finally the gyro-Bohm behaviour is provided by $T^{3 / 2}$, a positive dependence of $\chi_{\mathrm{e}}$ upon $T_{\mathrm{e}}$ which leads to an increase of stiffness with temperature. The instabilities can be suppressed by an adequate magnetic and velocity shear leading to the ITBs discussed in [1]. Such plasmas are not investigated in the present work. Theory indicates that for a comparison between devices of different size the dimensionless variable $R / L_{T_{\mathrm{e}}}$ with $1 / L_{T_{\mathrm{e}}}=\nabla T_{\mathrm{e}} / T_{\mathrm{e}}$ should be used.

For more details on the theoretical background and numerical calculations see to the two companion papers on transport theory presented at this conference [2,3].

The basic properties of these instabilities will be the guideline of the main part of this paper. In particular, the existence of the threshold $(\nabla T / T)_{\mathrm{c}}$ is obviously a key parameter which determines the temperature profiles. In the stiff case the temperature profiles are determined by equation (1) and by the boundary condition which is the edge temperature at the outer boundary of the stiff region, generally close to the plasma edge, or the pedestal region in the $\mathrm{H}$-mode. This means that the core temperature is proportional to the edge temperature, or alternatively that the temperature profiles plotted on a semi-log scale are shifted with respect to each other, according to their edge temperatures.

\subsection{Experimental requirements}

Transport studies can be carried out in quasi steady-state by power-balance analysis, yielding the usual transport coefficients. Modulation techniques are very useful in providing information on diffusion and convection, and on the resilience properties of the profiles. For all these studies, a good knowledge of the heating deposition profiles, as well as good measurement of temperature profiles (both of the ions and electrons because of coupling), density and $Z_{\text {eff }}$ are required. Separation between ion and electron channels is only possible when the energy exchange between the two species is not dominant. To study the electron transport specifically, particularly good conditions are provided by using preferentially dominant electron heating at low density. Electron heating can be provided by lower hybrid heating and current drive, fast wave in the ion cyclotron frequency domain (ICRH, FWEH) or electron cyclotron heating or current drive (ECH, ECCD). The electron cyclotron scenarios offer particularly attractive possibilities because this heating method provides a well defined pure electron heating profile. The single pass absorption is $100 \%$ for the $\mathrm{X}$-mode second harmonic scheme. The location of the power deposition can be easily varied by adjusting the magnetic field and mirror launchers. The width of the deposition profile can be quite narrow compared with the plasma cross section.

\subsection{Analysis of transport}

As recognized a long time ago, the plasma cross section in tokamaks can be divided into three regions relevant for transport studies, as follows.

- The 'central region': this is the plasma region around the magnetic axis, typically inside 
the $q=1$ surface or inside the mixing radius of the sawteeth. Transport in this part is believed to be mainly determined by the sawteeth in conventional discharges.

- The 'confinement region': outside the central region, we find the 'confinement region' which extends towards the plasma edge. There, the intrinsic transport of both heat and particles can be studied and this is where our paper is concentrated.

- The 'edge region': this part of the plasma is a narrow zone between the confinement region and the limiter or the separatrix. It is basically the pedestal in the H-mode plasmas. Transport in the edge region is dominated by the transport barrier and ELMs in H-modes and generally may be by convection, radiation losses and atomic physics. Describing transport in this part of the plasma is beyond the scope of this paper. However, as indicated above, this region is important because it provides the boundary condition for the profiles in the confinement region.

Transport can be studied in quasi steady state, on the confinement time scale. The power balance analysis yields, for the electrons, the heat diffusivity $\chi_{\mathrm{e}}^{\mathrm{PB}}$ according to the equation

$$
q_{\mathrm{e}}=-n_{\mathrm{e}} \chi_{\mathrm{e}}^{\mathrm{PB}} \nabla T_{\mathrm{e}}
$$

where $q_{\mathrm{e}}$ is the electron heat flux per surface unit, $n_{\mathrm{e}}$ the electron density and $T_{\mathrm{e}}$ the temperature. This analysis assumes that transport is purely diffusive as represented by $\chi_{\mathrm{e}}^{\mathrm{PB}}$. In some cases a convective term is added. In steady state diffusion and convection cannot be differentiated; a time evolution is required to allow the separation.

In perturbative experiments one investigates the response of a steady-state plasma to a small perturbation. Such perturbations can be provided by power modulation of the heating power, density modulation by the external gas valve, or injection of impurities by laser blow off. Some perturbations are provided by the plasma itself such as sawteeth or ELMs. In perturbative experiments (see the review [9] and references therein) the analysis can be carried out using the linearized diffusion equation which yields the perturbative diffusivity $\chi_{\mathrm{e}}^{\text {pert }}$ of electron heat transport.

$$
\chi_{\mathrm{e}}^{\text {pert }}=\chi_{\mathrm{e}}^{\mathrm{PB}}+\frac{\partial \chi_{\mathrm{e}}}{\partial \nabla T_{\mathrm{e}}} \nabla T_{\mathrm{e}} .
$$

In the concept of profile stiffness, the second term of the right-hand part of (3) can be high for $\nabla T_{\mathrm{e}} / T_{\mathrm{e}}>\left(\nabla T_{\mathrm{e}} / T_{\mathrm{e}}\right)_{\mathrm{c}}$ and $\chi_{\mathrm{e}}^{\mathrm{pert}}$ is in general larger than $\chi_{\mathrm{e}}^{\mathrm{PB}}$. When the perturbation is periodic the modulated data can be extracted by Fourier transform and $\chi_{\mathrm{e}}^{\text {pert }}$ is then deduced from the phase and amplitude using a slab model, [9]. Corrections for cylindrical geometry and density gradient can be taken into account [10]. In this method, diffusion and convection can be separated: the phase is insensitive to convection whereas the amplitude reacts to convection with a magnitude which decreases with frequency. The combination of both the power balance and the perturbative method is quite powerful in transport investigations as will be shown later. In particular, power modulation with ECH combined with a high-quality ECE diagnostic provides ideal conditions for such studies.

\section{Experimental transport results in the central region}

As mentioned above, the central region is the plasma zone influenced by the sawteeth in conventional scenarios. Transport in this region is basically determined by these MHD instabilities which regularly expel energy and particles. Sawteeth are believed to keep the $q$ profile quite close to one in the region inside the $q=1$ surface, providing a flat magnetic shear profile. In RTP [11] (see figure 1(a)), a transport barrier is observed near the $q=1$ surface which may be correlated to the shear profile. This barrier, clearly visible on the $T_{\mathrm{e}}$ 

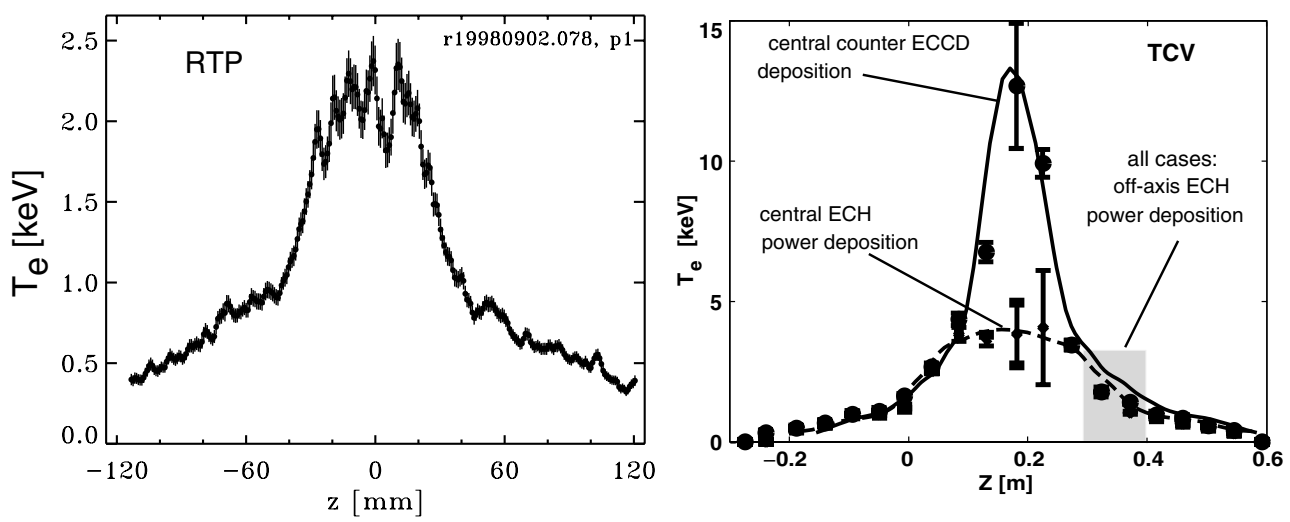

Figure 1. $T_{\mathrm{e}}$ profiles for in the central region in RTP and TCV, both with on-axis ECH or counterECCD. The filaments can be clearly seen in RTP as well as the barrier at $q=1$. The TCV profiles illustrate the effect of counter-ECCD leading to very high temperatures. (Courtesy of Beurskens [13] for RTP and Angioni for TCV [19].)

profile (figure 1) has also been confirmed by modulation experiments with ECH in RTP [11]. A barrier, located somewhat outside $q=1$, has also been observed in TCV, using poloidal sweeps of the ECH deposition [12]. Figure 1(a) for RTP also shows, inside the barrier, the so-called filaments which are strong $T_{\mathrm{e}}$ variations on a centimetre radial scale [13]. These structures, only observed inside the $q=1$ surface, are believed in RTP to have an $m=1 / n=1$ topology and to require flat shear. They have a lifetime much shorter than the confinement time and are only visible with the ultrahigh-resolution Thomson scattering in RTP [14]. With this diagnostic filaments have also been seen in the stellarator TJ-II [15] and in TEXTOR [16, 17]. Similar phenomena were claimed to be observed in TEXT with the ECE diagnostic and are attributed to the $m=1 / n=1$ precursor of the sawteeth [18] in the presence of central ECH. Independently of the physics interpretation, filaments are most probably able to drive a transport in the central region.

The suppression of sawteeth generally only provides a moderate increase of confinement in the central region. Adding counter-ECCD in this region causes the electron temperature to peak strongly as shown in TCV (figure 1(b)), leading to the ICEC regime [19]. This regime does not require the suppression of the sawteeth.

\section{Experimental transport results in the confinement region}

\subsection{On-axis heating}

4.1.1. Steady-state studies. It was observed in the 1980s [20-23] and more recently [24-26], first in ohmic discharges and later with rather centrally deposited auxiliary heating, that generally the electron temperature profiles were very similar. This feature seems to be almost independent of the experimental conditions and has been called profile 'consistency', 'resilience' or 'stiffness'. Most recently, motivated by the models based on ITG and TEMs or ETG physics predicting constant $\nabla T / T$ values, temperature profiles have been plotted on a semi-log scale and indeed exhibited the expected properties: similar shape with constant $\nabla T / T$ and shifted with respect to the edge temperature, see for instance [24, 25, 27] in $\mathrm{H}$-modes with rather equilibrated ion and electron heating at medium density and [28] in L-modes with dominant electron heating at low density. Interestingly, more than one decade earlier, TFTR 
Table 1. Overview of the data from six tokamaks of figure 2, ordered by increasing size.

\begin{tabular}{|c|c|c|c|c|c|c|}
\hline Tokamak & $\begin{array}{l}R \\
(\mathrm{~m})\end{array}$ & Shape/config. & $\begin{array}{l}B_{T} \\
(\mathrm{~T})\end{array}$ & $\begin{array}{l}\bar{n}_{\mathrm{e}} \\
\left(10^{19} \mathrm{~m}^{-3}\right)\end{array}$ & Heating & $\begin{array}{l}\text { Power } \\
(\mathrm{MW})\end{array}$ \\
\hline RTP & 0.72 & circ./lim. & 2 & 3 & $\mathrm{ECH}$ & 0.4 \\
\hline TCV & 0.88 & variable & 1.4 & 1.5 & $\mathrm{ECH}$ & 2.7 \\
\hline FTU & 0.93 & circ./lim. & 5.5 & 10 & $\mathrm{ECH}$ & 0.8 \\
\hline AUG & 1.65 & elong./div. & 2.3 & 2 & $\mathrm{ECH}$ & 1.6 \\
\hline TS & 2.30 & circ./lim. & 2.2 & 4 & FWEH & 7.0 \\
\hline JET & 3.0 & elong./div. & 2.8 & 4 & ICRF D(H) & 4.0 \\
\hline
\end{tabular}

electron temperature profiles had already been plotted in semi-log diagrams exhibiting the same behaviour [20,23].

In specific studies of electron transport with dominant electron heating the same properties were found in all the tokamaks, as illustrated in figure 2 by six examples. In ASDEX Upgrade, RTP, FTU and TCV the heating method was ECH in L-mode plasmas. In JET [29] ICRH minority combined with NBI was used in H-modes, whereas in Tore Supra [30] fast wave electron heating was applied. The experimental parameters are summarized in table 1.

The wide range in size, from $0.72 \mathrm{~m}$ in RTP to $3.0 \mathrm{~m}$ in JET, must be underlined. Note also the wide range in density and magnetic field for devices of almost the same size, FTU, RTP and TCV. In FTU ions and electrons are strongly coupled which is not the case in RTP or TCV. Despite these quite different experimental parameters, the $T_{\mathrm{e}}$ profiles in these power scans clearly exhibit the almost constant value of $\nabla T_{\mathrm{e}} / T_{\mathrm{e}}$ in the confinement region $(0.3 \leqslant \rho \leqslant 0.8)$ and the shift of the profiles with the edge temperature. Moreover, the values of $R / L_{T_{\mathrm{e}}}$ at mid-radius in these devices is quite comparable, between 8 and 12 .

This appears clearly in the overview of the values of $R / L_{T_{\mathrm{e}}}$ taken at mid radius in the tokamaks shown in figure 3.

In this figure a representative average has been taken for each device. The deviation from this value is typically $\pm 20 \%$ for different plasma parameters. The weak trend with $R$ appearing in this figure is not believed to be real, but possibly reflects a weak decrease of $R / L_{T_{\mathrm{e}}}$ with density, which however might be due to variations of the electron and ion heat fluxes. In this respect note the low value for FTU which has the highest density. The actual cause of the variation of the $R / L_{T_{\mathrm{e}}}$ values is still under investigation. These consistent observations indicate that in conventional scenarios ( $\mathrm{L}$ or $\mathrm{H}$ modes) electron heat transport physics in the confinement region is very likely the same in all the tokamaks. Moreover, this strongly suggests that electron heat transport is driven by instabilities with quite similar $\left(R / L_{T_{\mathrm{e}}}\right)_{\mathrm{c}}$, as discussed in section 5 .

Following equation (1) a comparison between experiment and theory can be achieved in plotting the experimental $\chi_{\mathrm{e}}^{\mathrm{PB}}$ normalized by the gyro-Bohm factor $T_{\mathrm{e}}^{3 / 2}$, as was done in $[27,28]$. Results from four tokamaks are available and are compared in figure 4. All four machines clearly show a strong increase of electron transport above a threshold in $R / L_{T_{\mathrm{e}}}$, keeping the profile to a rather constant value of $R / L_{T_{\mathrm{e}}}$ over a major fraction of the plasma radius. In such plots, going from low to high values of $\nabla T_{\mathrm{e}} / T_{\mathrm{e}}$ corresponds to a radial excursion from the centre towards the edge. The precise shape of the curve depends on how $\nabla T_{\mathrm{e}}$ was taken. Taken along the major radius on the low field side of the plasma yields a monotonic curve whereas taken averaged over the magnetic surface yields an S-shaped curve. This is shown in the plot for ASDEX Upgrade where the grey diamonds illustrate the latter case for one power level. The overlap of the curves corresponding to the different power levels in ASDEX Upgrade and Tore Supra is a strong indication for the gyro-Bohm behaviour. The 

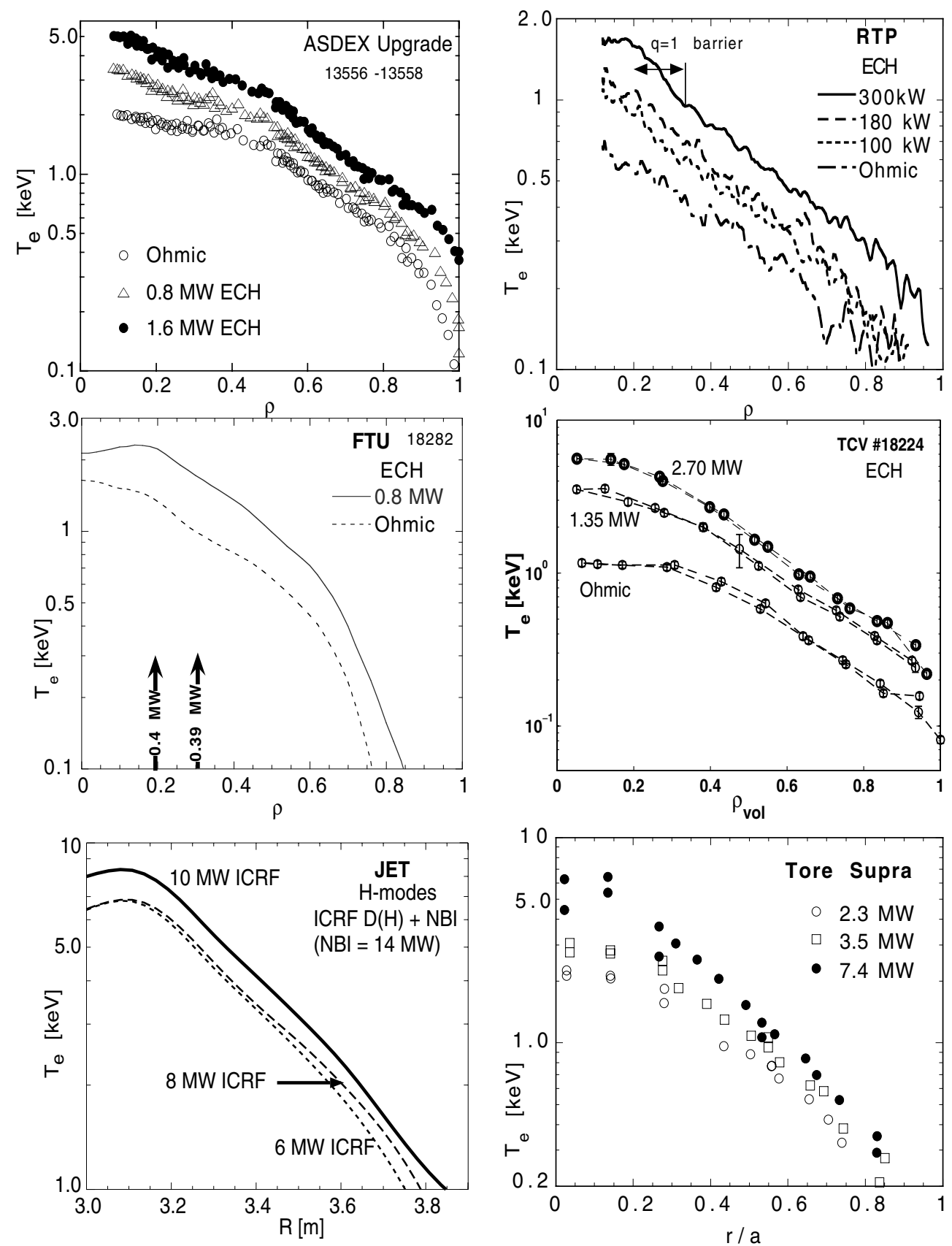

Figure 2. $T_{\mathrm{e}}$ profiles for central heating for ASDEX Upgrade, RTP, FTU TCV, JET and Tore Supra The regions with constant slope corresponding to constant $\nabla T_{\mathrm{e}} / T_{\mathrm{e}}$ can be clearly seen. (Courtesy of Mantica and Hogeweij for RTP, Jacchia for FTU, Angioni for TCV, Hoang for Tore Supra and Suttrop for JET.)

agreement is not as good in TCV. In ASDEX Upgrade the measurement of $R / L_{T_{\mathrm{e}}}$ was achieved with a high precision using a radial scan of the plasmas [28].

As seen in figure 2 , towards the edge the $T_{\mathrm{e}}$ profiles deviate from the almost constant 


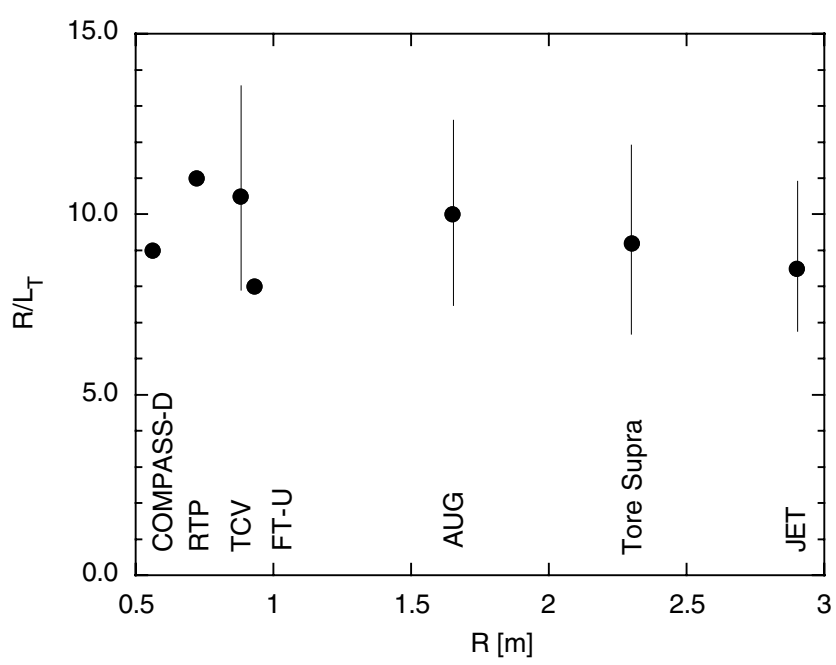

Figure 3. Values of $R / L_{T_{\mathrm{e}}}$ for different tokamaks. The error bars indicate the variation when available. Note: the data were collected and discussed during a workshop on electron transport held at IPP on the 18th of April 2001.

$R / L_{T_{\mathrm{e}}}$ and become steeper. The responsible physical mechanism is not yet really identified. It could be a complete loss of stiffness at low temperatures, typically below $700 \mathrm{eV}$, as suggested by ASDEX Upgrade and Tore Supra analyses $[28,30]$ or an increase of $\left(\nabla T_{\mathrm{e}} / T_{\mathrm{e}}\right)_{\mathrm{c}}$ at the plasma edge. Again, this behaviour was also observed in TFTR with the same turn-off temperature [23].

For JET a similar analysis for the profiles of figure 2 yields somewhat different results which are still under investigation [29]. The difference might be due to the stronger ion heat flux and coupling between electrons and ions.

All these results under quite different plasma conditions clearly demonstrate that the profile resilience is a quite general property of electron transport.

During the 1990s, extensive investigations of electron transport with ECH were carried out in RTP. They indicated the existence of several transport barriers linked with the main rational surfaces and attributed to the locally flattened magnetic shear there. The strongest barrier is located at the $q=1$ surface as indicated above. These RTP results have been described in detail in several publications and, for a more complete discussion, we refer the reader to the corresponding literature, [11,31-34]. They lead to an empirical transport model which consists of a series of transport barriers (low transport) distributed over the plasma radius and separated by zones of high transport [32]. More recent investigations in RTP with modulated ECH attribute the lower transport in the barriers not to a reduction of diffusion but rather to an inwardly-directed convection localized around the rational surface [35]. This effect is believed to be related to the magnetic shear shape in these narrow regions. However, it could not be determined experimentally whether or not $\mathrm{ECH}$ is required at this very location to create the convective effect.

Apart from the barrier near $q=1$, those related to other rational $q$ surfaces are weak or absent in the case of central heating. Therefore, the experimental observations in RTP are not in contradiction with the hypothesis that transport is governed by critical gradient physics, because this mechanism may well determine the heat transport in the regions between the barriers. This is supported by figure 2 for RTP. 

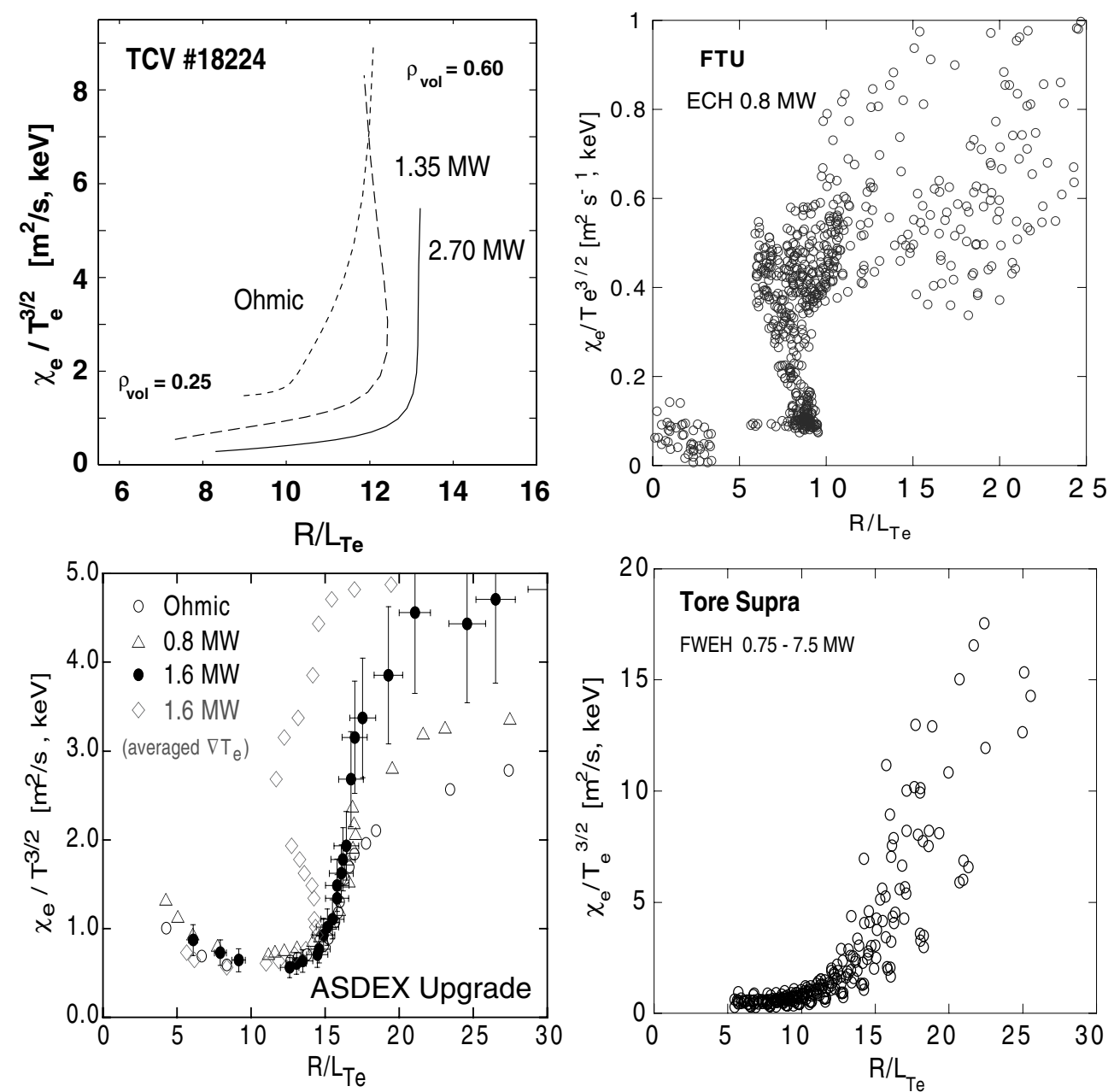

Figure 4. Normalized heat diffusivity versus $R / L_{T_{\mathrm{e}}}$ for ASDEX Upgrade, FTU and TCV with $\mathrm{ECH}$ and Tore Supra with fast wave electron heating.

4.1.2. Perturbative studies with on-axis heating. Perturbative studies are an important complement to these steady-state analyses. The values of $\chi_{\mathrm{e}}^{\text {pert }}$ deduced from heat pulses propagating from the centre towards the edge can be compared with $\chi_{\mathrm{e}}^{\mathrm{PB}}$. Numerous investigations have been done in the past based on the propagation of sawtooth heat pulses. The analysis generally yields $2 \leqslant \chi_{\mathrm{e}}^{\text {pert }} / \chi_{\mathrm{e}}^{\mathrm{PB}} \leqslant 15$. Power modulation experiments with $\mathrm{ECH}$ in RTP yielded similar results [36]. In this work an off-set linear scaling for $q_{\mathrm{e}}$ was invoked. This is equivalent to assuming the existence of $\left(\nabla T_{\mathrm{e}} / T_{\mathrm{e}}\right)_{\mathrm{c}}$. It has been shown recently in ASDEX Upgrade that both in $\mathrm{L}$ and $\mathrm{H}$ modes and independently of the plasma isotope (hydrogen or deuterium), $\chi_{\mathrm{e}}^{\text {pert }} / \chi_{\mathrm{e}}^{\mathrm{PB}}$ for the outward propagating pulses from $\mathrm{ECH}$ and sawteeth increases with $T_{\mathrm{e}}$ [37]. This is consistent with the increase of stiffness with $T_{\mathrm{e}}$ due to the $T_{\mathrm{e}}^{3 / 2}$ in equation (1). Moreover, the finite temperature offset found for $\chi_{\mathrm{e}}^{\text {pert }} / \chi_{\mathrm{e}}^{\mathrm{PB}}=1$ is a clear indication of a threshold in transport as $\nabla T_{\mathrm{e}}$ and $T_{\mathrm{e}}$ are closely related. The ratio $\chi_{\mathrm{e}}^{\text {pert }} / \chi_{\mathrm{e}}^{\mathrm{PB}}$ can reach values as high as 20 for ECH pulses and 30 for sawtooth pulses. These experimental observations from RTP and ASDEX Upgrade are clearly in agreement with the 

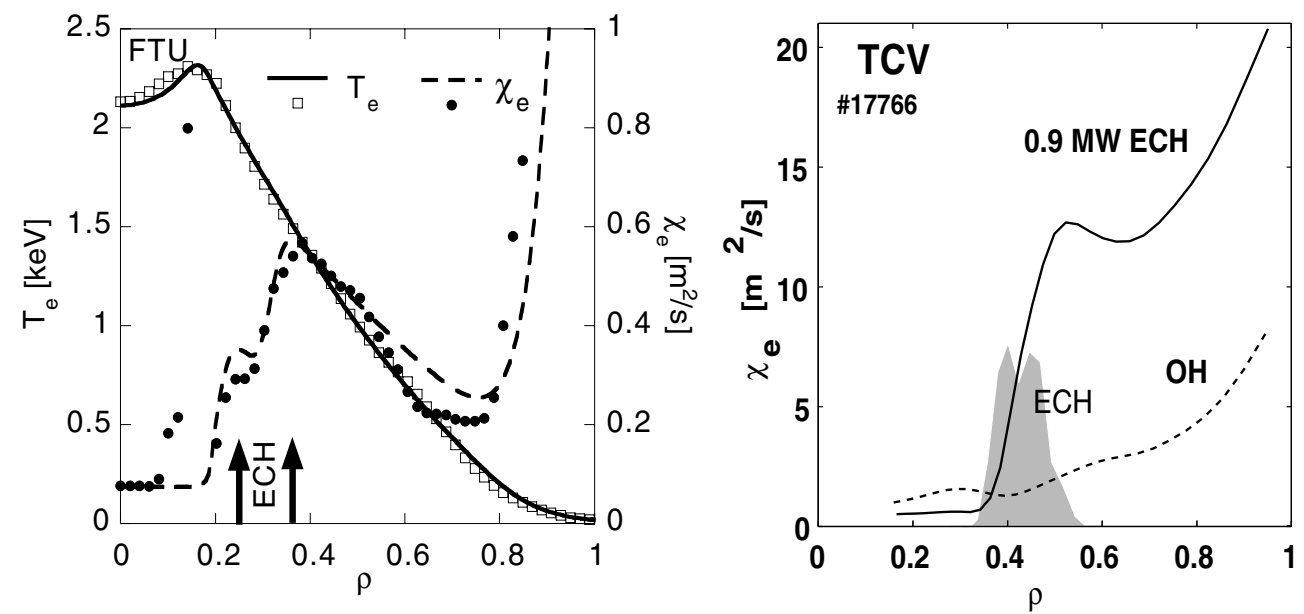

Figure 5. $\chi_{\mathrm{e}}^{\mathrm{PB}}$ profiles in FTU and TCV for off-axis cases with ECH as indicated in each plot. For FTU the points are the data and the curves are the modelling based on equation (1) as explained in the text. For TCV the curves are from power balance analysis and the ECH deposition from TORAY calculations. (Courtesy of Cirant and Jacchia [44] for FTU and Angioni for TCV.)

concept of profile stiffness for which large values of $\chi_{\mathrm{e}}^{\text {pert }} / \chi_{\mathrm{e}}^{\mathrm{PB}}$ may be expected, as explained in section 2 .

\subsection{Off-axis heating}

The profile resilience has motivated several experiments using off-axis heating in order to investigate the transport behaviour in the region with reduced heating power inside the deposition, with NBI [22, 38, 39] and with ECH [21, 40-42]. These experiments showed that the temperature profiles generally remained rather peaked in the centre, requiring a very low transport there. Recent off-axis experiments dedicated to electron transport studies have been performed: with ICRH in JET [29]; with ECH in ASDEX Upgrade [27]; FTU [43-46]; and in TCV.

This is illustrated by a recent example from FTU in figure 5 for a moderately off-axis case. The curves represent modelling based on equation (1) which shows that assuming a critical gradient length is necessary to reproduce the data [44].

Here also, the $T_{\mathrm{e}}$ profiles remain rather peaked and a very low transport is yielded by the power balance in the central region inside the power deposition. Due to the quite low power in the volume inside the power deposition, one expects a low transport. However, crossing the heating deposition zone, the transport represented by $\chi_{\mathrm{e}}^{\mathrm{PB}}$ varies strongly for a small variation of the plasma parameters such as $q, T_{\mathrm{e}}, \nabla T_{\mathrm{e}}, n_{\mathrm{e}}$. The drop in electron heat conductivity in the inner region is much larger than that expected from a conservative hypothesis such as $\chi_{\mathrm{e}} \propto \nabla T_{\mathrm{e}}^{\alpha}$ or $\chi_{\mathrm{e}} \propto T_{\mathrm{e}}^{\alpha}$ with $\alpha \leqslant 2$. Therefore this clearly shows that a threshold behaviour of $\chi_{\mathrm{e}}$ is required to explain the experimental data. The values of $\chi_{\mathrm{e}}^{\mathrm{PB}}$ can be as low as the neoclassical diffusion and even become negative in some cases reported in $[43,44]$ similarly to earlier observations in DIII-D [41]. In the cases where the power balance yields a negative value for $\chi_{\mathrm{e}}$ in the region inside the deposited power, an inward directed convection term $U$ can be assumed to bring $\chi_{\mathrm{e}}$ back to a just positive value. As transport is very low in this region anyway, the required value for $U$ is of the order of 1 to $2 \mathrm{~m} \mathrm{~s}^{-1}$ [44], which is very small 
and of the order of the Ware pinch in this region. It must be underlined that in the presence of sawteeth, convection can be caused by the MHD phenomena. In the cases where sawteeth could be avoided by an adequate current profile, the experimental errors remain large but the results are claimed to be outside the uncertainties. It should be emphasized that such conditions might be favourable for the formation of the filaments described in previously and it is not excluded that, like MHD phenomena, they cause convection.

4.2.1. Modulation experiments with off-axis heating. Detailed modulation experiments in the presence of off-axis ECH have been carried out in ASDEX Upgrade [27,28]. The flexible ECH system composed of four independent injection lines allows the experimental questions to be addressed with great flexibility. The main results, which show without ambiguity that electron transport is governed by a critical gradient physics, are summarized in this section. In a first set of experiments, two ECH lines (called $\mathcal{C}$ ) were depositing up to $800 \mathrm{~kW} \mathrm{CW}$ off-axis at $\rho_{\text {dep }} \approx 0.5$. This determines two regions of transport: the 'inner region $\mathcal{I}$ ' inside the ECH deposition and the 'outer region $\mathcal{O}$ ' outside of the deposition. According to the power balance results described in the previous section, these two regions are expected to be in quite different transport regimes: below $\left(\nabla T_{\mathrm{e}} / T_{\mathrm{e}}\right)_{\mathrm{c}}$ in region $\mathcal{I}$ and above $\left(\nabla T_{\mathrm{e}} / T_{\mathrm{e}}\right)_{\mathrm{c}}$ in region $\mathcal{O}$. Simultaneously, using another ECH line $\mathcal{M}$, modulated at $30 \mathrm{~Hz}$, heat pulses were launched from the edge $(\rho \approx 0.8)$ towards the centre. The analysis of the propagation of these pulses shows clearly the two transport regions: in region $\mathcal{O}$ the propagation is fast with $\chi_{\mathrm{e}}^{\text {pert }} / \chi_{\mathrm{e}}^{\mathrm{PB}}$ up to 10 whereas in region $\mathcal{I}, \chi_{\mathrm{e}}^{\text {pert }} / \chi_{\mathrm{e}}^{\mathrm{PB}}$ is close to unity. In these experiments the behaviour of phase and amplitude does not yield any indication of convection. Varying the radial position of the deposition and the power of lines $\mathcal{C}$ indicates that the separation between regions $\mathcal{I}$ and $\mathcal{O}$ is indeed linked with the ECH deposition from lines $\mathcal{C}$ and that the difference in transport between regions $\mathcal{I}$ and $\mathcal{O}$ depends on the heating power delivered by lines $\mathcal{C}$.

An overview of the results is given in figure 6(a)-(c) for the $800 \mathrm{~kW}$ case. It shows the amplitude and phase of the heat pulses in the presence of power from $\mathcal{C}$ compared with the case without this power (figures $6(\mathrm{a})$ and (b)). The corresponding values of $\chi_{\mathrm{e}}^{\mathrm{PB}}$ and $\chi_{\mathrm{e}}^{\text {pert }}$ are shown in figure $6(\mathrm{c})$, the latter being as high as $\approx 10 \mathrm{~m}^{2} \mathrm{~s}^{-1}$ in the stiff region and extremely low in the non-stiff region. The increase of $\chi_{\mathrm{e}}^{\text {pert }}$ with heating power of $\mathcal{C}$ or equivalently with temperature is shown in figure $6(\mathrm{~d})$. This is in qualitative agreement with the increase of stiffness with $T_{\mathrm{e}}$ provided in equation (1) by $T_{\mathrm{e}}^{3 / 2}$.

Profile stiffness can be characterized quantitatively by the ratio $\chi_{\mathrm{e}}^{\text {pert }} / \chi_{\mathrm{e}}^{\mathrm{PB}}$, i.e. the 'stiffness factor'. If it is close to unity the profiles are not stiff, but stiff profiles would exhibit a value clearly larger than unity. This factor has been studied experimentally in ASDEX Upgrade as a function of $\nabla T_{\mathrm{e}} / T_{\mathrm{e}}$.

Here again, heat pulses were launched from the plasma edge towards the centre by the ECH line $\mathcal{M}$ depositing at $\rho \approx 0.9$. Their propagation was analysed in a narrow region around $\rho=0.6$. The changes of $\nabla T_{\mathrm{e}} / T_{\mathrm{e}}$ in the region of analysis were achieved by varying the power of the lines $\mathcal{C}$ having the position of their absorption layer at $\rho_{\mathrm{dep}}=0.5$ or $\rho_{\mathrm{dep}}=0.7$. In the configuration with $\rho_{\text {dep }}=0.5$ the measurement at $\rho \approx 0.6$ is placed in the stiff region of the profile, whereas in the case $\rho_{\text {dep }}=0.7$ it is in the region where the profile is driven to values below $\left(\nabla T_{\mathrm{e}} / T_{\mathrm{e}}\right)_{\mathrm{c}}$. The results, given in figure $7(\mathrm{a})$, show a threshold in $\nabla T_{\mathrm{e}} / T_{\mathrm{e}}$ with a strong increase of $\chi_{\mathrm{e}}^{\mathrm{HP}}$ by about one order of magnitude for a small variation in $\nabla T_{\mathrm{e}} / T_{\mathrm{e}}$, whereas the variation of $\chi_{\mathrm{e}}^{\mathrm{PB}}$ is smaller. The corresponding stiffness factor shown in figure 7(b) clearly exhibits a strong increase above $\left(\nabla T_{\mathrm{e}} / T_{\mathrm{e}}\right)_{\mathrm{c}} \approx 6 \mathrm{~m}^{-1}$, demonstrating without ambiguity the transition from non-stiff to stiff $T_{\mathrm{e}}$ profiles when crossing $\left(\nabla T_{\mathrm{e}} / T_{\mathrm{e}}\right)_{\mathrm{c}}$. 

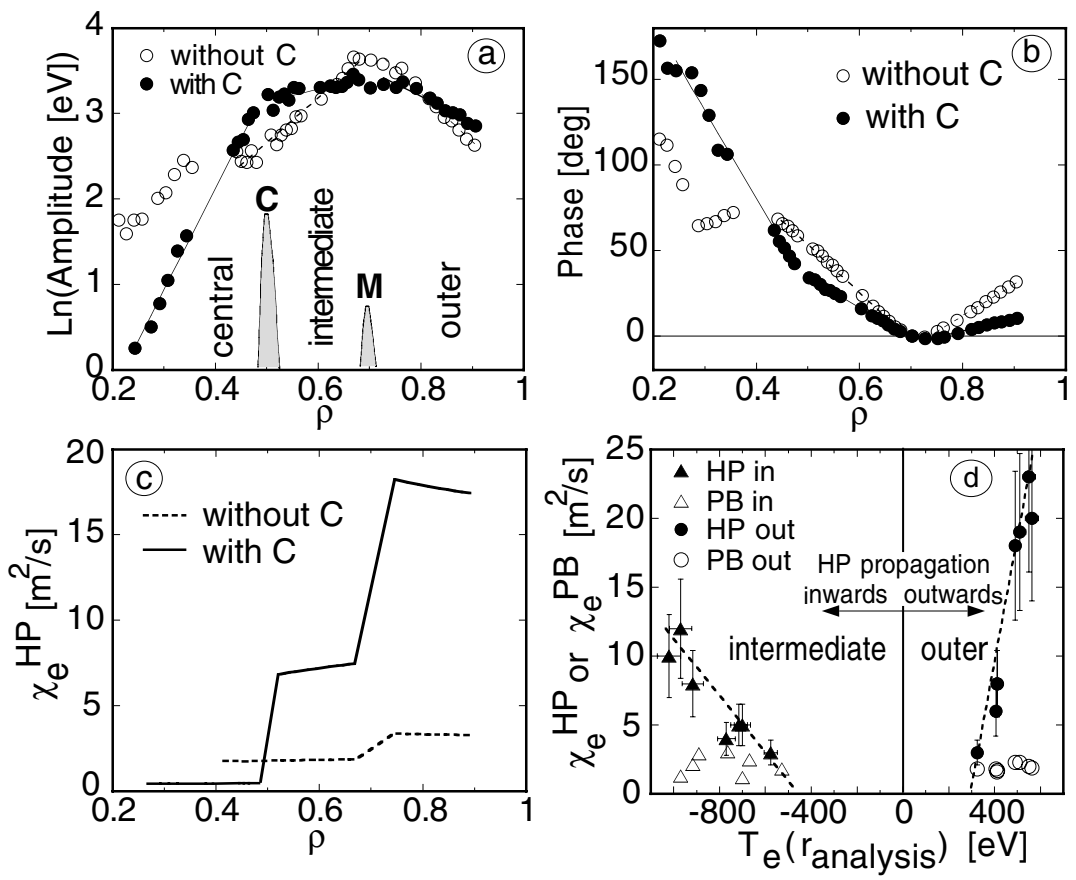

Figure 6. ECH modulation experiments in ASDEX Upgrade with and without power at mid-radius from lines $\mathcal{C}$. The flat gradients of amplitude and phase indicate the fast propagation of the heat pulses (large $\chi_{\mathrm{e}}^{\text {pert }}$ ) and the steep gradients the slow propagation (low $\chi_{\mathrm{e}}^{\text {pert }}$ ).
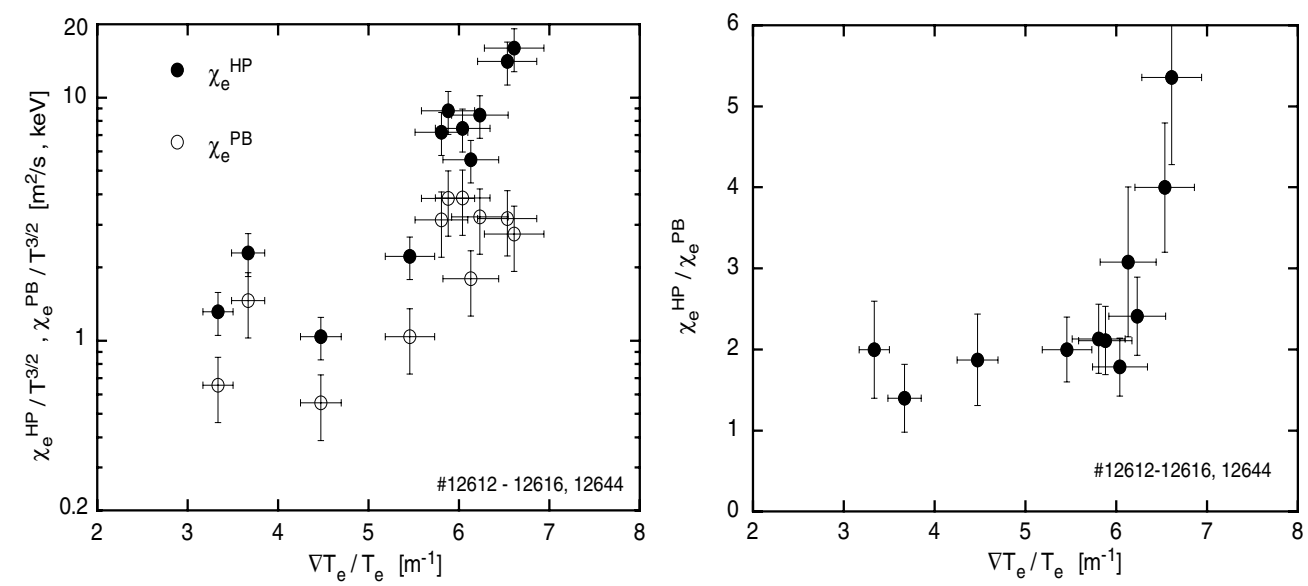

Figure 7. $\chi_{\mathrm{e}}^{\mathrm{HP}}$ and $\chi_{\mathrm{e}}^{\mathrm{PB}}$ measured at $\rho=0.6$ plotted versus $\nabla T_{\mathrm{e}} / T_{\mathrm{e}}$. The relative uncertainties on $\nabla T_{\mathrm{e}} / T_{\mathrm{e}}$ are small as this quantity is deduced from the same two ECE channels for all the points. The absolute (systematic) error is larger $(\approx 30 \%)$ but only causes a translation of all the points and therefore does not change the essence of the results.

\section{Comparison between experiment, theory and modelling}

A complete comparison of the experimental results with theory is beyond the scope of this paper. However, a brief summary is essential in an attempt to better understand the physics of electron 


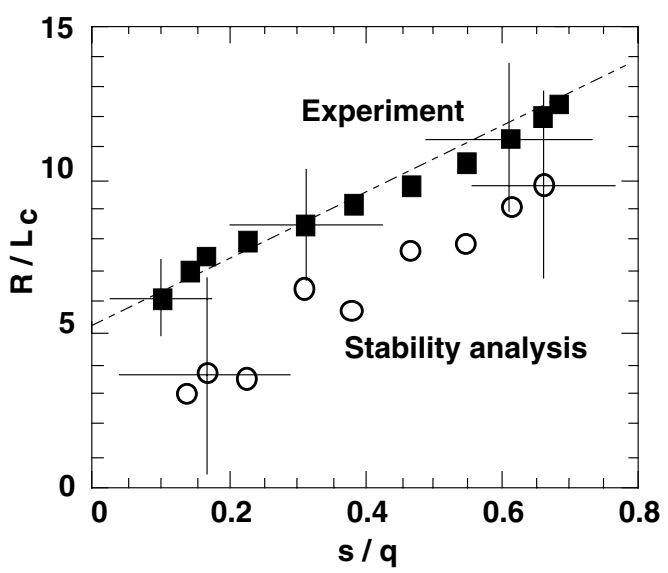

Figure 8. The dependence of the experimental values of $\left(1 / L_{T_{\mathrm{e}}}\right)_{\mathrm{c}}$ in Tore Supra versus $\hat{s} / q$. (Courtesy of Hoang [49].)

heat transport in tokamaks. Two kinds of models are considered: empirical and physicsbased. The former, in which the coefficients are adjusted to the experiments, includes physical hypotheses in a rather simple form and is quite useful for a comprehensive interpretation of the experimental observations. For the topic addressed here, models assuming the existence of a critical gradient length have been used for ASDEX Upgrade [47] and FTU [44]. They are basically of the form of equation (1). Both models are quite similar and confirm that the assumption of a threshold in $\nabla T_{\mathrm{e}} / T_{\mathrm{e}}$ is indeed required to provide a good agreement with the data. In the case of ASDEX Upgrade both steady state and ECH modulation described in the previous section could be modelled quite well without re-adjusting the parameters between the steady state and modulated cases. In the two devices, these studies indicate that $\left(\nabla T_{\mathrm{e}} / T_{\mathrm{e}}\right)_{\mathrm{c}}$ is lower than the $\nabla T_{\mathrm{e}} / T_{\mathrm{e}}$ of the experimental profiles. Therefore the profiles correspond to a working point above the threshold.

A model based on electromagnetic ETG physics has been successfully compared with the Tore Supra data [30,48]. In particular, the experimental $\hat{s} / q$ dependence of $\left(1 / L_{T_{\mathrm{e}}}\right)_{\mathrm{c}}$ shown in figure 8 from [49] agrees with the ETG model described in [48] and the formula for the ETG threshold given in [8].

It must be emphasized however, that the value of $\left(\nabla T_{\mathrm{e}} / T_{\mathrm{e}}\right)_{\mathrm{c}}$ is lower than that deduced from the experimental results. Stability studies indicate that TEMs are expected to be the dominant modes [49].

Physics-based models derived from first principles were also used to model ECH modulation experiments in DIII-D [50] and ASDEX Upgrade [51]. The experiments in ASDEX Upgrade were dedicated to electron transport studies at low density with dominant electron heating whereas those in DIII-D were in NBI-heated L-modes and also addressed ion transport. Both studies indicate that models based on ITG/TEM physics provide good agreement with the data. Concerning the electrons, the best agreement was provided in ASDEX Upgrade by the Weiland model [52], as shown in figure 9 for a case with moderately off-axis heating.

This figure shows excellent agreement for the modulated electron temperature for frequencies from the fundamental up to the fourth harmonic. This is a strong indication that under these conditions transport is governed by TEM modes. The agreement for the steady-state profiles, not shown here, is also very good. It is interesting to note that $\left(\nabla T_{\mathrm{e}} / T_{\mathrm{e}}\right)_{\mathrm{c}}$ is lower than $\left(\nabla T_{\mathrm{e}} / T_{\mathrm{e}}\right)$ of the $T_{\mathrm{e}}$ profiles by a factor of about two. This is in agreement with 


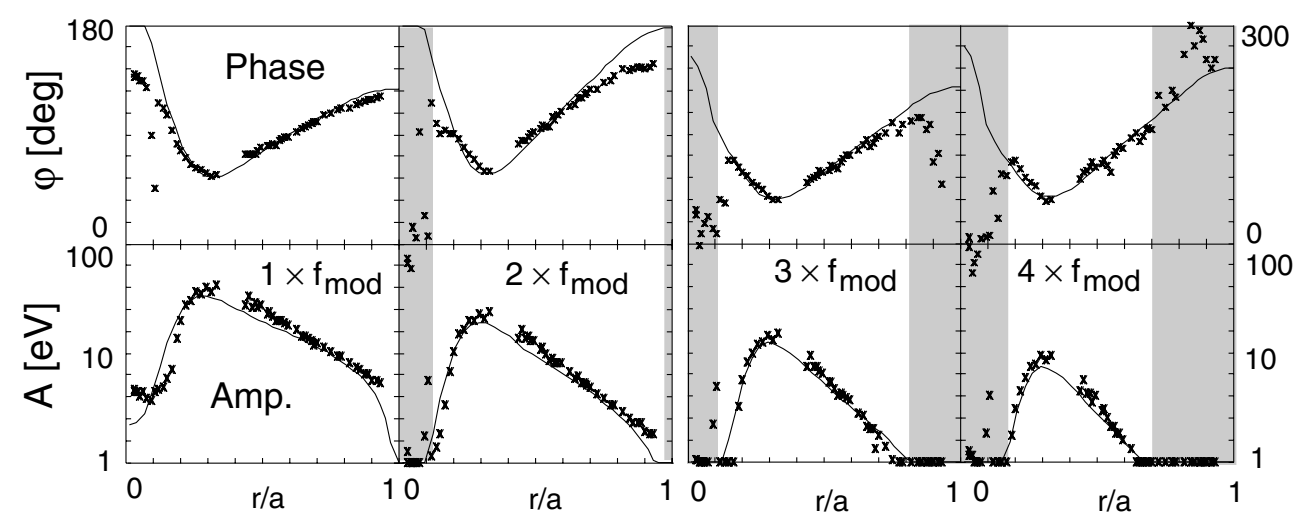

Figure 9. A comparison of modelling with the Weiland ITG/TEM model (curves) with phase and amplitude of the $T_{\mathrm{e}}$ perturbation induced by ECH modulation at about $30 \mathrm{~Hz}$. The grey zones mark the regions where the signal-to-noise ratio is poor.

the findings from empirical modelling and shows that the $T_{\mathrm{e}}$ profiles are not very stiff. As the threshold $\left(R / L_{T_{\mathrm{e}}}\right)_{\mathrm{c}}$ for TEMs varies little from device to device, this is consistent with the observation that $R / L_{T_{\mathrm{e}}}$ is similar in all the tokamaks considered here (figure 3 ). At present, there is no transport model based on ETG turbulence with streamers which would allow a comparison with the above results. It must be noted that, due to its parameter dependences [8], in particular the factor $Z_{\text {eff }} T_{\mathrm{e}} / T_{\mathrm{i}}$, the value of $\left(R / L_{T_{\mathrm{e}}}\right)_{\mathrm{c}}$ for ETG is more subjected to variations with experimental parameters. In particular, in the low density cases with high $T_{\mathrm{e}} / T_{\mathrm{i}}$ values achieved with ECH in ASDEX Upgrade, RTP or TCV, the theoretical value of $\left(R / L_{T_{\mathrm{e}}}\right)_{\mathrm{c}}$ for ETG is above the experimental values of $R / L_{T_{\mathrm{e}}}$ and the ETG turbulence is not expected to be excited. However, it is not excluded that TEM and/or ETG turbulence govern the electron heat transport depending on the parameters and plasma regions. Finally, the interplay between electrons and ions through coupling and ITG/TEM instabilities has to be further investigated, as it is clearly an important player in the determination of the actual value of $\nabla T_{\mathrm{e}} / T_{\mathrm{e}}$.

\section{Other aspects}

Other aspects of electron transport could not be addressed in detail in this paper and are briefly touched on here.

Scaling experiments of $\rho^{*}$ have shown the gyro-Bohm behaviour of the electron transport. This is in agreement with the experiments reported here, as the $T^{3 / 2}$ factor of equation (1) provides a good normalization.

The non-local effects, which occur in the very low density range of tokamak operation, are not yet satisfactorily explained. Therefore non-local studies should be continued in dedicated experiments, in particular with the aim of a comparison with the critical gradient hypothesis.

The relation between electron heat transport and magnetic or density fluctuations has been investigated and shows a correlation. A reduction of transport is linked with a reduction of the fluctuation level. Fluctuation measurements addressing specifically TEM and ETG turbulence including streamers might greatly help in discriminating between the two possibilities.

A correlation between the electron heat and particle transport has been observed for a long time. This effect seems to be particularly strong with ECH as shown by the 'density pump out', e.g., in the stellarator W7-AS [53]. In fact, in all the ECH heated experiments 
described in this paper, the density reacts to electron heating; the density profiles become flat with central ECH. This is a consequence of temperature profile resilience if a constant ratio between heat and particle transport is assumed [54]. Such a model yields, without caseto-case adjustment, a quite good agreement with experimental observations in very different situations. Further investigations of possible particle transport by TEM and ETG turbulence would certainly improve the understanding in this field. The flat and even hollow density profiles observed in TCV are interpreted as a consequence of the $m / n=1 / 1=1$ mode and the loss of axisymmetry [55].

\section{Conclusion}

Electron transport exhibits many different features which are not all due to pure turbulent transport, but can be caused by other mechanisms, for instance MHD-like events. In the confinement region, believed to be dominated by turbulent transport, all the tokamaks show a quite similar behaviour of $T_{\mathrm{e}}$ profiles and electron transport. The studies in steady state with on-axis and off-axis heating, supported by empirical and physics-based modelling, strongly suggest that electron heat transport is governed by turbulence with a threshold in $\nabla T_{\mathrm{e}} / T_{\mathrm{e}}$. This is in agreement with the two theoretically-expected candidates, TEM and ETG modes. This leads to a nonlinear behaviour in the region of the threshold and to a similarity of the $T_{\mathrm{e}}$ profiles above the threshold, for on-axis heating. This is confirmed by recent modulation experiments with ECH and is in agreement with previous findings from transient transport. It must be underlined that generally the working point is clearly above $\left(\nabla T_{\mathrm{e}} / T_{\mathrm{e}}\right)_{\mathrm{c}}$ and the $T_{\mathrm{e}}$ profiles are not very stiff. Within the clear similarity of the shape a certain room for variation exists, depending on the operational conditions. In particular the coupling to the ions, which seem to have a stiffer behaviour, could play an important role, which remains to be investigated in detail. The actual mechanism(s) TEM or ETG has not been unambiguously identified yet. Further experimental work is needed in dedicated experiments, for instance combining simultaneously specific scenarios with dominant electron heating and identification of the turbulence properties by fluctuation measurements. In addition, the continuation of inter-machine comparison will certainly yield fruitful information. Some common experiments and analyses are foreseen. The expansion of ECH systems will significantly contribute to the progress in this field.

\section{Acknowledgments}

It is a pleasure to thank colleagues from the different institutes who kindly supplied information about published work: S Cirant, J C DeBoo, T Fukuda and Valovič. Fruitful discussions with X Garbet, F Imbeaux, F Jenko and A G Peeters are warmly acknowledged. Finally, C Angioni, S Cirant, A Jacchia, A Kendl, J Lister, O Sauter, P Mantica, J Stober, W Suttrop, G Tardini and H Weisen who contributed to the 'Satellite Workshop on Electron Transport' held at IPP in April 2001, are warmly thanked, as well as the Heads of the Associations who made their participation possible.

\section{References}

[1] Barbato E 2001 Plasma Phys. Control. Fusion 43 A289

[2] Garbet X 2001 Plasma Phys. Control. Fusion 43 A253

[3] Jenko F 2001 Plasma Phys. Control. Fusion 43 A141

[4] Romanelli F et al 1989 Plasma Phys. Control. Fusion 311535

[5] Jenko F et al 2000 Phys. Plasmas 71904 
[6] Dorland W et al 2000 Phys. Rev. Lett. 855579

[7] Nilsson J et al 1995 Nucl. Fusion 35497

[8] Jenko F et al 2001 Phys. Plasmas 84096

[9] Lopes Cardozo N J 1995 Plasma Phys. Control. Fusion 37799

[10] Jacchia A et al 1991 Phys. Fluids B 33033

[11] Lopes Cardozo N et al 1997 Plasma Phys. Control. Fusion 39 suppl. 12B, B303

[12] Henderson M et al 2000 Workshop on Strong Microwaves (Nizhny Nogvorod, 1999) ed A G Litvak vol 1 p 114

[13] Beurskens M et al 2001 Plasma Phys. Control. Fusion 4313

[14] Barth C et al 1997 Rev. Sci. Instrum. 683380

[15] Herranz J et al 2000 Phys. Rev. Lett. 854715

[16] Schüller C et al 2000 EPS 2000 (Budapest) post deadline paper

[17] Schüller C et al 2001 Proc. 28th EPS Controlled Fusion and Plasma Physics (Funchol, 2001) Europhys. Conf. Abstracts Or.01

[18] Porcelli F et al 1999 Phys. Rev. Lett. 821458

[19] Pietrzyk Z et al 2001 Phys. Rev. Lett. 861530

[20] Goldston R et al 1987 Plasma Phys. Contr. Fus. Res., Proc. 11th IAEA Conf. (Kyoto, 1986) vol 3 p 75

[21] Alikaev V et al 1987 Plasma Phys. Contr. Fus. Res., Proc. 11th IAEA Conf. (Kyoto, 1986) vol 3 p 111

[22] Wagner F et al 1986 Phys. Rev. Lett. 562187

[23] Taylor G et al 1989 Nucl. Fusion 293

[24] Suttrop W et al 1997 Plasma Phys. Control. Fusion 392051

[25] Horton L D et al 1999 Plasma Phys. Control. Fusion 41 B329

[26] Weisen $\mathrm{H}$ et al 1999 Phys. Plasmas 61

[27] Ryter F et al 2001 Nucl. Fusion 41537

[28] Ryter F et al 2001 Phys. Rev. Lett. 865498

[29] Suttrop W et al these proceedings, paper P3.016

[30] Hoang G T et al 2000 Plasma Phys. Contr. Fus. Res., Proc. 18th IAEA Conf. (Sorrento, 2000)

[31] Hogeweij G et al 1996 Nucl. Fusion 36535

[32] Hogeweij G et al 1998 Nucl. Fusion 381881

[33] Mantica P et al 1999 Phys. Rev. Lett. 825048

[34] de Baar M et al 1999 Phys. Plasmas 61355

[35] Mantica P et al 2000 Phys. Rev. Lett. 854534

[36] Mantica P et al 1996 Nucl. Fusion 361317

[37] Ryter F et al 2001 Phys. Rev. Lett. 862325

[38] Schissel D P et al 1992 Nucl. Fusion 32689

[39] Gohil P et al 1998 Nucl. Fusion 38425

[40] Luce T et al 1992 Phys. Rev. Lett. 6852

[41] Petty Cet al 1994 Nucl. Fusion 34121

[42] Hogeweij G et al 1996 Phys. Rev. Lett. 76632

[43] Sozzi C et al 2000 Plasma Phys. Contr. Fus. Res., Proc. 18th IAEA Conf. (Sorrento, 2000) Paper EXP5/13

[44] Jacchia A et al 2001 Topical Conf. Radio Frequency Power in Plasmas (Oxnard, USA, 2001)

[45] Cirant S et al 2001 Topical Conf. Radio Frequency Power in Plasmas (Oxnard, USA, 2001)

[46] Sozzi C et al 2001 Proc. 28th EPS Controlled Fusion and Plasma Physics (Funchol, 2001) Europhys. Conf. Abstracts P3.059

[47] Imbeaux F et al 2001 Plasma Phys. Control. Fusion 431503

[48] Horton W et al 2000 Phys. Plasmas 71494

[49] Hoang G T et al Phys. Rev. Lett. submitted

[50] DeBoo J et al 1999 Nucl. Fusion 391935

[51] Tardini G et al 2001 Nucl. Fusion submitted

[52] Nordman H et al 1990 Nucl. Fusion 30983

[53] Hartfuss H J et al 1994 Plasma Phys. Control. Fusion 36 B17

[54] Stober J et al 2001 Plasma Phys. Control. Fusion 43 A39

[55] Weisen $\mathrm{H}$ et al 2001 Nucl. Fusion 411227 